\title{
Torsional Dynamics of Steerable Needles: Modeling and Fluoroscopic Guidance
}

\author{
John P. Swensen*, Member, IEEE, MingDe Lin, Member, IEEE, Allison M. Okamura, Fellow, IEEE, \\ and Noah J. Cowan, Senior Member, IEEE
}

\begin{abstract}
Needle insertions underlie a diversity of medical interventions. Steerable needles provide a means by which to enhance existing needle-based interventions and facilitate new ones. Tip-steerable needles follow a curved path and can be steered by twisting the needle base during insertion, but this twisting excites torsional dynamics that introduce a discrepancy between the base and tip twist angles. Here, we model the torsional dynamics of a flexible rod-such as a tip-steerable needle-during subsurface insertion and develop a new controller based on the model. The torsional model incorporates time-varying mode shapes to capture the changing boundary conditions inherent during insertion. Numerical simulations and physical experiments using two distinct setups-stereo camera feedback in semitransparent artificial tissue and feedback control with real-time X-ray imaging in optically opaque artificial tissue-demonstrate the need to account for torsional dynamics in control of the needle tip.
\end{abstract}

Index Terms-Continuum robot, medical robot, robot dynamics, tip-steerable needles.

\section{INTRODUCTION}

A CCURATE placement of a needle tip is crucial to the success of many interventional and diagnostic procedures. For interventions ranging from the delivery of localized treatment to millimeter-accuracy sampling of tissues, improvement in the accuracy of needle tip placement has the potential to improve patient outcomes and reliability of diagnosis. Such clinical needle interventions remain remarkably unchanged in delivery mechanism since Alexander Wood's 19th century idea: deliver therapy subcutaneously through the lumen of sharp, stiff tubes [1]. Most existing needle insertion procedures are dominated by two century-old paradigms: 1) insertion is accomplished manually by a clinician and 2) the needle is a rigid steel tube. However,

Manuscript received November 13, 2013; revised March 17, 2014; accepted May 6, 2014. Date of publication May 21, 2014; date of current version October 16, 2014. This work was supported by the National Institutes of Health under Grant R01-EB006435. Asterisk indicates corresponding author.

*J. P. Swensen is with the Department of Mechanical Engineering and Materials Science, Yale University, New Haven, CT 06520, USA (e-mail: john.swensen@yale.edu).

M. Lin is with the Clinical Informatics, Interventional, and Translational Solutions, Philips Research North America, Briarcliff Manor, NY 10510, USA (e-mail: ming.lin@philips.com).

A. M. Okamura is with the Department of Mechanical Engineering, Stanford University, Stanford, CA 94305, USA (e-mail: aokamura@ stanford.edu).

N. J. Cowan is with the Department of Mechanical Engineering, Johns Hopkins University, Baltimore, MD 21218, USA (e-mail: ncowan@jhu.edu).

This paper contains supplemental material available online at http://ieeexplore.ieee.org (File size: $27 \mathrm{~KB}$ ).

Color versions of one or more of the figures in this paper are available online at http://ieeexplore.ieee.org.

Digital Object Identifier 10.1109/TBME.2014.2326161 in the last decade, researchers have begun to explore new methods of needle insertion, needle insertion assistance tools for clinicians, and fully automated needle insertion robots [2]-[7].

Continuous fluoroscopic imaging provides feedback to the clinician during manual needle-based interventions such as biopsies, ablations, and device placement (i.e., stents, drains, GI/GU tubes, and periradicular therapy). A needle insertion robot, similar to the one used in this paper for needle insertions, could automate this process, thereby improving targeting accuracy while limiting radiation exposure to the clinician. Targeting accuracy is particularly important in the lymphatic system, which often require several needle insertions using current techniques, as well as in nearly all musculoskeletal section procedures, such as bone tumor ablations, cyst aspirations, and periradicular therapy [8]-[10]. Significant benefit could also be derived in renal procedures, prostate biopsies, and insertion of afterloading catheters [11]-[14]. Targeting accuracy is also essential for neurosurgical procedures, such as targeting the ventricles of the brain to drain fluid in patients with elevated intracerebral pressure and spinal procedures [15], [16]. The potential for steerable needle robots to assist in achieving high accuracy in needle placement leads us to the development of improved models and associated control techniques for a specific needle-steering setup as a first step toward this goal.

In this paper, we develop new dynamic models of flexible needles with associated control algorithms for the automatic control of tip-steerable needles under fluoroscopic guidance, and demonstrate system efficacy through simulation and experiments. We derive a model for the torsional dynamics of a needle in tissue that is "twisted" at its base, and couple the dynamics with an existing kinematic model of tip-steerable needles [6], [17]. The primary purpose of the additional modeling of torsion is to improve the estimation and control of the needle tip; here, we perform control to a plane during continuous insertion to facilitate comparison with previous work [18]. Most previous models, motion planners, and control algorithms for tip-steerable needles assume that the insertion and rotational velocities at the tip of the needle are equivalent to those at the base of the needle [6], [17]-[20]. However, to achieve the desired bending inside tissue, tip-steerable needles are typically made of the superelastic alloy nickel-titanium (Nitinol). These superelastic needles severely violate the assumption that base and tip torsional velocities are equal: twisting a long slender beam from the controlled proximal end generally results in "torsional windup" along the length of the needle [21]. This realization motivates our development herein of a system model that incorporates the torsional dynamics, so that an automatic feedback 
controller can be designed to compensate accordingly. In addition to simulations, we present experimental results of the proposed method using both stereo-image feedback and fluoroscopy feedback in artificial tissues.

The work of the past decade on nontraditional needle insertion methods can be classified into several categories including alternative needle design, needle control and planning, and modeling needle/tissue interactions. These works identify the significant potential that steerable needles have to change the landscape of percutaneous therapies into a "post Wood era." DiMaio and Salcudean [4] investigates the effects of tissue deformation, needle cutting, and external manipulation on robotically controlled needles in artificial tissues. Okazawa et al. [5] designed a mechanism for affecting needle motion inside tissue by engineering the needle itself; their design consisted of an outer straight tube and inner stylet with a preset fixed radius of curvature used to cause bending during needle insertion. Webster et al. [6] and Park et al. [17] modeled needles with beveled tips as a nonholonomic kinematic systems and tested this model in surrogate tissues. Further work shows that this nonholonomic model may be valid for other types of tip-steerable needles including those with a precurved section at the tip of the needle [22].

Our goal is to improve modeling and control for tip-steerable needles that are actuated outside of the body at the proximal end (or base) of the needle [6], [17]. Combinations of other needle steering methodologies, such as transverse and "tip/tilt" motion outside the tissue [23], or manipulation of the tissue itself [24], might ultimately be used in a single, integrated setup, as proposed in [25] (see Fig. 2 therein). However, this paper focuses on the torsional dynamics of the needle with only twisting and insertion. In any similar system with high-torsional compliance and active torsional loading, torsional dynamics-like those considered in this paper-will play a critical role in tooltip motion. The models and controllers presented in this paper provide a model of torsional dynamics that can be used for continuous needle insertion under real medical imaging modalities to help move the current state of the art for steerable needles from laboratory to clinic, a goal that still has yet to be accomplished on any large scale.

Previous methods of automatic feedback control for tipsteerable needles by Kallem and Cowan [18] conducted closedloop control to a virtual plane inside the tissue via feedback linearization, assuming no torsional dynamics. They showed that the controlled behavior of the needles deviated from the theoretical predictions in a way that suggested torsional dynamics may be significant. Reed et al. [21] confirmed the significance of needle torsion on needle dynamics, and developed a model of torsional dynamics for a fixed length of needle inside the tissue and performed open-loop torsional compensation during large needle tip reorientations. The work presented in this paper builds on these two results, developing new models for continuous-insertion, length-varying torsional dynamics inside and outside the tissue, as well as the feedback control algorithms necessary to achieve control of the tip to a desired plane within the tissue during continuous insertion. Several other researchers have conducted experiments where the needle motion is paused while imaging [18], [23], [25], [26]; however, any time the nee- dle motion is stopped, static friction between needle and tissue becomes a significant and uncertain factor. By continuously inserting the needle for lengths longer than a few millimeters to $1 \mathrm{~cm}$, the making and breaking of static friction and its associated effect on uncertainty of the position and orientation of the needle tip can be avoided to a great degree, thus simplifying modeling and control greatly. There are also other ongoing efforts for steerable needle control and robotically controlled straight-needle insertions under alternative imaging modalities such as ultrasound [24], [27] and MRI-compatible robots [28][30]. To date, the majority of needle insertion research has been conducted in a laboratory with visible light cameras and semitransparent tissue surrogates. Herein, we have conducted experiments involving continuous insertion with automatic feedback from fluoroscopic imaging.

Other work incorporates needle and needle-tissue dynamics, but these have typically focused on tissue deformation and have approached the modeling problem using finite-element methods [4] or simplified "virtual spring" models [23]. Here, we neglect tissue deformation as it is unlikely to cause a significant deviation in human tissue [21], and focus our modeling effort on needle torsional wind-up, including the viscous drag between needle and tissue. Our approach uses a proper orthogonal decomposition, coupled with a Galerkin projection [31], [32], allowing us to side-step finite-element techniques altogether. This approach gives us an alternative infinite-dimensional representation of the hypothesized system dynamics, which can then be systematically reduced to a manageable form by truncating higher order terms in the infinite-dimensional expansion, whereby the error between the truncated system and the infinitedimensional system can be made arbitrarily small through the choice of truncation order. Our model-which can be applied quite generally to flexible, linear-elastic rods being inserted at a constant velocity into a substrate-is similar to a time-varying modal approach used for approximating the linear vibrational dynamics of a spacecraft antenna as it is extended [33].

Initial results related to this study were presented in a conference paper [34], which we expand upon significantly in the present paper with alternative formulation for torsional dynamics, dimensional analysis, and experiments under fluoroscopy feedback. After reviewing the basic tip-steering kinematic model developed by Webster et al. [6], Section II describes our new time-varying torsional model. We then provide a new integrated model of needle steering that effectively concatenates the torsional dynamics with tip-steering kinematics. Section III describes the strategy used to control the tip of the needle to a plane by compensating for the torsional dynamics. Section IV shows simulations and experiments demonstrating the efficacy of the method, with comparisons between control using models with and without torsion compensation. Section V concludes with some comments about the methods, observations about the experiments, and proposed directions for future research.

\section{MODELING}

The motion of the needle is composed of two parts: a kinematic description of needle tip motion based on body-fixed 
(a)

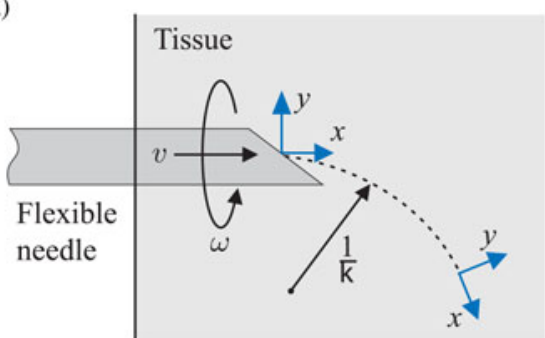

(b)

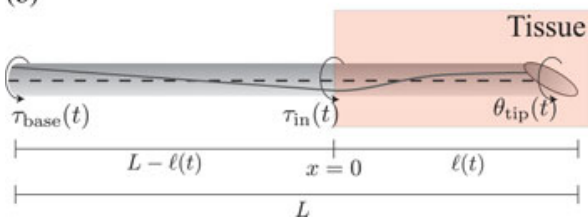

Fig. 1. Model of steering dynamics. (a) Kinematic model used to represent the insertion of tip-steerable flexible needles, adapted from [6]. As the needle is inserted at velocity $v$, a bevel or other tip asymmetry causes a lateral force that deflects the needle along a curved path of radius $1 / \kappa$. Rotations about the tip with angular velocity $\omega$ reorient the bevel tip. (Modified with permission from [35].) (b) Time-varying modal dynamics formulation for the portion of the needle inside the tissue and an ideal torsional spring for the portion outside the tissue. There is a time-varying boundary condition as the needle is inserted, based on the changing length of the needle inside the tissue. Here, the dashed line represents an imaginary line inscribed on the surface of the needle with zero torsion. The gray line indicates the same inscribed line while the needle is under torsion.

velocity vector fields and a dynamic description of the torsional motion along the shaft of the needle.

\section{A. Kinematics of Tip-Steerable Needles}

Given the body-fixed frame at the tip of the needle, as shown in Fig. 1(a), the kinematic model developed by Webster et al. [6] describes the motion of the needle using the bicycle model with a fixed turning radius; we now review this model and establish notation. The insertion velocity at the tip of the needle prescribes the forward velocity of the bicycle, and the rotational velocity at the tip changes the orientation of the plane in which the bicycle travels. This model can be succinctly described by the left-invariant vector field describing the motion of the needle tip in its body-fixed frame. The rigid body transformation

$$
g=\left[\begin{array}{cc}
R & \mathrm{~d} \\
0^{T} & 1
\end{array}\right] \in S E(3)
$$

describes the orientation and position of the needle tip with respect to an inertial frame, where $R \in S O(3)$ and $\mathrm{d} \in \mathbb{R}^{3}$ are the rotation matrix and tip position, respectively. The velocities in the body fixed frame are given as

$$
\Omega_{\mathrm{tip}}=\left(g^{-1} \dot{g}\right)^{\vee}=V_{1} v+V_{2} \omega
$$

where the twists associated with needle insertion velocity and tip rotational velocity are $V_{1}=\kappa e_{3}+e_{4}$ and $V_{2}=e_{6}$. Most previous work on tip-steerable needles assumes that the insertion and rotational velocities at base and tip are equal such that $v=u_{1}$ and $\omega=u_{2}$, where $\left(u_{1}, u_{2}\right)$ are the insertional and rotational control inputs to the needle insertion robot. However, the rotational input, $u_{2}$ twists the proximal end of a long slender rod embedded in tissue, and the tissue provides significant frictional torque, so the rotation at the base only couples to the rotation at the tip through an intervening viscoelastic dynamical system. Reed et al. [21] demonstrate the need for torsional dynamics compensation, but their solution for torsion compensation assumes that the needle remains a fixed length inside the tissue, an assumption clearly violated during continuous needle insertion. We build on Reed et al. result in the next section by incorporating the time-varying boundary conditions that result from continuous needle insertion.

\section{B. Torsional Dynamic Model}

The dynamic model accounts for torsion both inside and outside the tissue as shown in Fig. 1(b). We model the portion inside the tissue using a partial differential equation (PDE) that incorporates rotational inertial effects, viscous drag forces between needle and tissues, and linear elastic shear forces due to torsional deformation. We model the portion outside the tissue as an ideal torsional spring whose spring constant is a function of the polar moment of inertia $J$, the needle shear modulus $G$, and the length outside the tissue $L-\ell(t)$, where $L$ is the entire needle length and $\ell(t)$ is the portion of the needle inside the tissue.

Using the Newton-Euler formulation for an infinitesimal portion of the needle inside the tissue, Reed et al. [21] derived a PDE in $\theta(x, t)$ a function of space and time

$$
\underbrace{\rho J}_{\eta} \frac{\partial^{2} \theta}{\partial t^{2}}+\beta \frac{\partial \theta}{\partial t}-\underbrace{J G}_{\kappa} \frac{\partial^{2} \theta}{\partial x^{2}}=\delta(x) \tau_{\text {in }}(t)
$$

where $\beta$ represents viscous damping (assumed to be uniform along the needle shaft), $\eta$ is rotational inertia, $\kappa$ is the torsional stiffness, $\rho$ is the density of the needle material, $J$ is the polar moment of inertia of the needle, and $G$ is the shear modulus of the needle material. In Reed et al. model, it is assumed that the external torque on the needle is applied at the point where the needle enters the tissue, which is incorporated mathematically via the product of a spatial Dirac impulse function, $\delta(x)$, and the torque at the coupling between motor and the needle, $\tau_{\text {in }}(t)$.

Henceforth, our derivations deviate significantly from the previous work done by Reed et al. [21] in the following two key respects: 1) we no longer model the system as a fixed length inside the tissue and 2) we do not assume torque control at the point the needle enters the tissue. The primary modeling task going forward is to transform the formulation of torsional dynamics given by the PDE given in (3) into a system of ordinary differential equations that are amenable to estimation and control techniques. In what follows, we perform this transformation and put the result into a useful form amenable to implementation.

First, we note that the torque at the motor-needle interface is exactly the same as the torque at the tissue boundary, i.e., $\tau_{\text {in }}=\tau_{\text {base }}$, since we assume an ideal torsional spring for the portion of the needle outside the tissue. Hence, for the portion of the needle inside the tissue, we can still adopt the formulation in (3), where the spatial impulse is taken at $x=0$. In other words, 
the torsional angle of the needle at the point of insertion into the tissue can be different from the angle of the motor shaft, but the torque is the same all along the portion of the needle outside the tissue.

Because the needle length inside the tissue is changing, we cannot make the assumption that the modal solution is separable in both space and time. Instead, we can only assume that the mode shapes are functions of both space and time and the mode coefficients are functions of time

$$
\theta(x, t)=\frac{1}{2} q_{0}(t)+\sum_{k=1}^{\infty} \psi_{k}(x, t) q_{k}(t)+\sum_{k=1}^{\infty} \phi_{k}(x, t) p_{k}(t)
$$

where

$$
\left.\begin{array}{l}
\psi_{j}(x, t)=\cos \left(\omega_{j}(t) x\right) \\
\phi_{j}(x, t)=\sin \left(\omega_{j}(t) x\right)
\end{array}\right\}, \quad \text { and } \quad \omega_{j}(t)=\frac{j \pi}{\ell(t)} .
$$

The basis for the proper orthogonal decomposition includes the torsional modes along the length of the needle as given in (5). This system with the time-varying length inside the tissue does not separate in space and time and we must perform the Galerkin projection and verify that each projection results in an ODE in time only; fortunately, the Galerkin projection for this system indeed results in a time-domain ODE. To perform our Galerkin projection, we first substitute (4) into (3), and then project onto each mode shape. This rote computation is not included here in its entirety and we refer the reader to the accompanying digital resource for a complete derivation of the model written for Mathematica (Wolfram Research, Champaign, IL, USA). The properties of key importance for simplification during the computations are related to the orthogonality conditions for the mode shapes of a Fourier series.

The appropriate number of torsional modes to include in the truncated approximation of the system depends significantly on the tissue properties, needle properties, and control scheme employed. In Section IV, we compare and contrast through simulation and experiments several model order truncations. Reed et al. analyzed a specific needle and tissue combination with experimentally computed damping and used Hankel singular values to determine an appropriate model order. The technique of Hankel singular values is complicated considerably in our formulation because the system is now a time-varying system for which an analytical form of the state transition matrix has not been obtained. For a general $n$th order projection, the resulting ODE takes the form

$$
\begin{aligned}
{\left[\begin{array}{cc}
M_{1} & 0 \\
0 & M_{2}
\end{array}\right]\left[\begin{array}{c}
\ddot{\mathbf{q}} \\
\ddot{\mathbf{p}}
\end{array}\right] } & +\left[\begin{array}{cc}
D_{1} & 0 \\
0 & D_{2}
\end{array}\right]\left[\begin{array}{c}
\dot{\mathbf{q}} \\
\dot{\mathbf{p}}
\end{array}\right] \\
& +\left[\begin{array}{cc}
K_{1} & 0 \\
0 & K_{2}
\end{array}\right]\left[\begin{array}{l}
\mathbf{q} \\
\mathbf{p}
\end{array}\right]=\left[\begin{array}{c}
P \\
0
\end{array}\right] \tau_{\text {in }}
\end{aligned}
$$

where the vectors $\mathbf{q}$ and $\mathbf{p}$ are the time-varying coefficients to the cosine and sine modes, respectively, as given in (4). Here, $M_{i}$ are constant diagonal inertia matrices, $D_{i}$ are time-varying damping matrices, and $K_{i}$ are the time-varying stiffness matrices. The dynamical (6) is a truncated modal approximation of the exact torsional dynamics originally in (3); the purpose of this transformation is to facilitate estimation and control.

We first note that the cosine and sine modes are decoupled, the sine modes are unforced, and the sine modes are naturally asymptotically stable. We assume that for any experiment the sine modes are initially unexcited. Thus, since the sine modes are uncontrollable they remain at zero, and do not couple to the cosine modes, and hence, we reduce them out of the model and refer to the matrices associated with the cosine mode as $M, D$, and $K$ throughout the remainder of this paper for simplicity.

After some tedious calculations, the specific structure of these terms are as follows. The diagonal inertia matrix is given by

$$
M=J \rho I_{n \times n} .
$$

For the matrices for representing the viscous damping and shear forces, the coefficients of each entry are functions of the row and column $(i, j)$ within the matrix

$d(i, j)= \begin{cases}-\frac{4(-1)^{i-j}(j-1)^{2}}{(j-i)(i+j-2)}, & \text { if } j>i \\ \operatorname{sgn}(1-i)+\frac{\beta l(t)}{J \rho v}, & \text { if } i=j \\ \frac{4(-1)^{j-i}(j-1)^{2}}{(i-j)(i+j-2)}, & \text { if } j<i\end{cases}$

$$
[D]_{i j}=d(i, j) \frac{J \rho v}{\ell(t)}
$$

$$
\begin{aligned}
& k(i, j)= \begin{cases}0, & \text { if } i=1 \text { or } j=1 \\
\frac{2 \pi^{2} G(i-1)^{2}}{p v^{2}}-\frac{1}{3} \pi^{2}(i-1)^{2}+\frac{1}{2}, & \text { if } i=j>1 \\
-\frac{8(i-1)^{2}(j-1)^{2}(-1)^{i-j}}{(j-i)^{2}(i+j-2)^{2}}, & \text { if } i \neq j\end{cases} \\
& {[K]_{i j}=k(i, j) \frac{J \rho v^{2}}{\ell^{2}(t)} .}
\end{aligned}
$$

An accompanying digital resource provides for the rapid construction of these matrices using Matlab (The MathWorks, Inc., Natick, Massachusetts, USA). Thus, the model for an arbitrary number of modal states can be generated without requiring experience in reducing the associated PDEs.

We model the portion of the needle outside the tissue as an ideal torsional spring, namely

$$
\tau_{\text {in }}(t)=\frac{J G}{L-\ell(t)}\left(\theta_{\text {base }}-\theta_{\text {in }}\right) .
$$

Here, the rotation at the insertion point of the needle $\theta_{\text {in }}$ is written in terms of the mode shapes and mode coefficients

$$
\begin{aligned}
\theta_{\text {in }}=\left.\theta(x, t)\right|_{x=0} & \approx \frac{1}{2} q_{0}+\sum_{k=1}^{n} \psi(0, t) q_{k}(t) \\
& =\underbrace{\left[\begin{array}{cc}
\frac{1}{2} & 1 \cdots 1
\end{array}\right]}_{C_{0}} \mathbf{q} .
\end{aligned}
$$

The torque resulting from the rotational position at the base of the needle and the modal representation of the rotational position at the insertion, obtained by substituting (13) into (12), is

$$
\tau_{\text {in }}=-\frac{J G}{L-\ell(t)} C_{0} \mathbf{q}+\frac{J G}{L-\ell(t)} \theta_{\text {base }}
$$


TABLE I

PARAMETERS USED IN SIMULATIONS AND EXPERIMENTS

\begin{tabular}{ll}
\hline \hline Parameter & Value \\
\hline Density $(\rho)$ & $6.453 \frac{\mathrm{kg}}{\mathrm{m}^{3}}$ \\
Polar moment of inertia $(J)$ & $1.5962-14 \mathrm{~m}^{4}$ \\
Shear modulus $(G)$ & $1.841210 \mathrm{Pascals}$ \\
Viscous drag $(\beta)$ & $4.753-3 \mathrm{~N} \cdot \mathrm{s}$ \\
Radius of curvature $(1 / \kappa)$ & $0.073 \mathrm{~m}$ \\
\hline \hline
\end{tabular}

The Parameters $\rho$ and $J$ were taken from manufacturer specifications, while $G, \beta$, and $\kappa$ were determined experimentally.

where $\theta_{\text {base }}$ is the control input for subsequent control computations. The term $\frac{J G}{L-\ell(t)}$ can be thought of as the time-varying, lumped-parameter spring constant for the portion of the needle outside the tissue.

Substituting the torque constraint from (14) into the ODE representing the torsional dynamics inside the tissue in (6) and neglecting the sinusoidal modes, the full torsional dynamics from base to tip is

$$
\begin{aligned}
M \ddot{\mathbf{q}} & +D \dot{\mathbf{q}} \\
& +\left(K+\frac{J G}{L-\ell(t)} P C_{0}\right) \mathbf{q}=\frac{J G}{L-\ell(t)} P \theta_{\text {base }} .
\end{aligned}
$$

We also note that for the needle and tissue parameters during our experiments (see Table I), a nondimensionalization analysis of (15) given in Appendix A indicates that the inertial forces associated with this system are quite small relative to the damping, shear, and control forces such that we can simplify the system to a coupled first order system

$$
\begin{aligned}
\dot{\mathbf{q}}= & \underbrace{-D^{-1}\left(K+\frac{J G}{L-\ell(t)} P C_{0}\right)}_{A(t)} \mathbf{q} \\
& +\underbrace{D^{-1} \frac{J G}{L-\ell(t)} P \theta_{\text {base }} .}_{B(t)}
\end{aligned}
$$

In the nondimensional analysis, there are three $\Pi$-groups but only one coefficient remains after the process of nondimensionalizing. The nondimensional parameter that remains is the characteristic length of

$$
x_{c}=\frac{d^{4}}{\beta}\left(\frac{\pi}{32} \sqrt{\rho G}\right) \sim \frac{d^{4}}{\beta}
$$

indicating that the dynamic inertial effects will become significant as the needle diameter $d$ decreases or the viscous damping between needle and tissues $\beta$ increases (assuming the density and shear modulus of Nitinol remains constant).

The velocity at the tip of the needle in the torsional dynamics $\dot{\theta}(x, t)$ is equal to the body-fixed rotational velocity $\omega$ given in (2). Thus, we compute

$$
\theta_{\text {tip }}=\left.\theta(x, t)\right|_{x=\ell(t)} \approx \frac{1}{2} q_{0}+\sum_{k=1}^{n} \psi(\ell(t), t) q_{k}(t)
$$

$$
=\underbrace{\left[\begin{array}{ccccc}
\frac{1}{2} & -1 & 1 & \cdots & (-1)^{n-1}
\end{array}\right]}_{C_{\ell}} \mathbf{q}
$$

and differentiate this to obtain

$$
\omega=\dot{\theta}_{\text {tip }}=C_{\ell} \dot{\mathbf{q}}=\underbrace{C_{\ell} A(t)}_{C(t)} \mathbf{q}+\underbrace{C_{\ell} B(t)}_{D(t)} \theta_{\text {base }}
$$

such that the final system can be written in a familiar linear, time-varying (LTV) form

$$
\begin{aligned}
& \dot{\mathbf{q}}=A(t) \mathbf{q}+B(t) \theta_{\text {base }} \\
& \omega=C(t) \mathbf{q}+D(t) \theta_{\text {base }} .
\end{aligned}
$$

The system representation given in this torsional dynamical model allows for two modes of control input: 1) torque control and 2) base angle control. Because we model the portion of the needle outside the tissue as an ideal torsional spring, the torque at the point of insertion is proportional to the difference in angle between the base and the insertion points. The torque along the entire length of the needle outside the tissue is equivalent to the torque at the point of insertion. However, to implement a controller that closes the loop around torque with this model including the ideal torsional spring, we would need a torque sensor at the base of the needle. Thus, we chose to close the loop around the position, eliminating the extra cost of an accurate torque sensor.

\section{Integration: Full Needle Dynamics}

We now couple the torsional dynamics with the existing kinematic model. The velocity at the tip of the needle in the torsional dynamics $\dot{\theta}(x, t)$ is equal to the body fixed rotational velocity $\omega$ given in (2). The full coupled form of the system can be described as

$$
\begin{aligned}
\Omega_{\mathrm{tip}} & =V_{1} v+V_{2}(C(t) \mathbf{q}+D(t) u) \\
\dot{\mathbf{q}} & =A(t) \mathbf{q}+B(t) u .
\end{aligned}
$$

To compare controllers built on our general torsional model with previous methods, we choose local coordinates for the tip of the needle, namely Z-Y-X Euler angles $(\alpha, \beta, \gamma)$ for orientation and $(x, y, z)$ for position as in Kallem and Cowan [18]. Following Kallem and Cowan further, we define the control task to be controlling the tip of the needle to an arbitrary plane in the tissue. With this control objective in mind, we can choose the local coordinates $\mathbf{s}^{T}=\left[\begin{array}{llllll}x & y & z & \alpha & \beta & \gamma\end{array}\right]$ such that they are relative to the plane. That is, $(y, z)$ are the position of the needle tip projected to the plane, $x$ is the orthogonal distance from the plane, and $\alpha$ is the rotation of the needle about an axis orthogonal to the plane. The remaining parameters, $\beta$ and $\gamma$, represent the pitch away from the plane and the rotation about the tip of the needle, respectively. The velocities in local coordinates can be related to the velocities in the body-fixed frame via the appropriate Jacobian

$$
\dot{\mathbf{s}}=J^{-1}(\mathrm{~s}) \Omega_{\mathrm{tip}} .
$$


Coupled with the torsional dynamics, the system can be represented as

$$
\left[\begin{array}{c}
\dot{\mathbf{s}} \\
\dot{\mathbf{q}}
\end{array}\right]=\left[\begin{array}{c}
J^{-1}(s)\left(V_{1} v+V_{2}(C(t) \mathbf{q}+D(t) u)\right) \\
A(t) \mathbf{q}+B(t) u
\end{array}\right] .
$$

For the task of controlling to a plane, Kallem and Cowan showed that (21) can be further reduced by "throwing away" the states $(y, z, \alpha)$, since they do not couple into the states $(x, \beta, \gamma)$, and that $(x, \beta, \gamma)=(0,0,0)$ corresponds to the needle tip traveling in the desired plane [18]. Thus, we augment these three states with the torsional states, i.e., $\mathbf{r}=\left[\begin{array}{llll}x & \beta & \gamma & \mathbf{q}^{T}\end{array}\right]^{T}$. In these coordinates, the full needle model with reduced state and torsional dynamics included is

$$
\begin{aligned}
\dot{\mathbf{r}} & =\left[\begin{array}{c}
v \sin \left(r_{2}\right) \\
\kappa v \sin \left(r_{3}\right) \\
-v \kappa \cos \left(r_{3}\right) \tan \left(r_{2}\right)+C(t) \mathbf{r}_{4 \cdots n}+D(t) u \\
A(t) \mathbf{r}_{4 \cdots n}+B(t) u
\end{array}\right] \\
& =: f(\mathbf{r}, u, t) .
\end{aligned}
$$

Here, we have a nonlinear and time-varying system for which we investigate common control practices for dealing with these types of systems. In the following section, we devise a control strategy with the assumption that the orthogonal distance of the needle tip from the plane is our only measurement.

\section{Control With Time-Varying Torsional Dynamics}

Kallem and Cowan used feedback linearization to generate a system for which LQR/LQG control was implemented. Using the model in (23), feedback linearization of the complete system is not possible due to the manner in which the control input $u$ enters into the system. Any attempt to feedback linearize the kinematic states necessarily introduces a nonlinearity into the torsional states.

The approach we take here is to 1) linearize the system about the origin, 2) define a feedforward control to decouple the kinematic state from the torsional states, and 3) compute the infinite horizon LQR gain to optimally control the decoupled kinematic states to the plane. A key component in this control strategy is to show that the final control input ensures that the torsional state remains bounded for the duration of our insertion.

The linearization about the origin of the system representation given in (23) is

$$
\dot{\mathbf{r}}=\left[\begin{array}{cccc}
0 & v & 0 & 0 \\
0 & 0 & \kappa v & 0 \\
0 & -\kappa v & 0 & C(t) \\
0 & 0 & 0 & A(t)
\end{array}\right] \mathbf{r}+\left[\begin{array}{c}
0 \\
0 \\
D(t) \\
B(t)
\end{array}\right] u
$$

Let the control input be defined as

$$
u=\frac{1}{D(t)}\left(-C(t) \mathbf{r}_{4 \cdots n}+u_{2}(t)\right) .
$$

The first term decouples the kinematic and torsional states. This results in

$$
\dot{\mathbf{r}}=\left[\begin{array}{cccc}
0 & v & 0 & 0 \\
0 & 0 & \kappa v & 0 \\
0 & -\kappa v & 0 & 0 \\
0 & 0 & 0 & A(t)-\frac{1}{D(t)} B(t) C(t)
\end{array}\right] \mathbf{r}
$$

$$
+\left[\begin{array}{c}
0 \\
0 \\
1 \\
\frac{1}{D(t)} B(t)
\end{array}\right] u_{2}(t)
$$

The first three states of the decoupled system are controllable and we implement a controller of the form

$$
u_{2}(t)=\left[\begin{array}{llllll}
k_{1} & k_{2} & k_{3} & 0 & \cdots & 0
\end{array}\right] \mathrm{r}
$$

where the gain is from the infinite horizon LQR solution to minimize the cost function

$$
J=\int_{t_{0}}^{\infty} \mathbf{r}_{1 \ldots 3}^{T}(\tau) Q \mathbf{r}_{1 \ldots 3}(\tau)+R u_{2}^{2}(\tau) d \tau .
$$

Heretofore, we have assumed full state feedback, but in practice we are only able to measure a scalar output, the distance from the desired plane

$$
\begin{aligned}
y & =\left[\begin{array}{llll}
1 & 0 & \cdots & 0
\end{array}\right] \mathbf{r} \\
& =r_{1} .
\end{aligned}
$$

Fortunately, a straightforward calculation can be used to verify observability of the system analytically by reconstructing the state from the output and its first $n-1$ derivatives. So, in our simulations and experiments, we use a Kalman filter to estimate the states of the linearized system (24) with the measurement model given in (29), and apply state feedback on this estimate, as is standard practice.

\section{Simulation AND ExPERIMENTAL RESUlts}

We present three results: 1) simulations with varying number of states, 2) experimental results using stereo triangulation as the sensing modality in our testbed environment, and 3) experimental results using $\mathrm{C}$-arm fluoroscopy as the sensing modality. In all cases, the needle and tissue parameters are based on the needle parameters given in Table I, with effort to match needle parameters given in [18] to facilitate performance comparisons, and semitransparent soft plastisol (M-F Mfg Co, Inc. stock \# $2228 \mathrm{LP}$ and $2228 \mathrm{~S}$ with plastic to softener ratio of 32:9) as a tissue surrogate.

Here, we present simulations and experiments to demonstrate that the modeling and control methods described herein provide an improvement over existing control methods that do not compensate for torsional dynamics during insertion. In lieu of a systematic approach for model reduction (e.g., based on the Hankel singular values) for this LTV system, we compared control efficacy based on models of different orders. Simulations were conducted using the same system parameters as the real system, with simulated process and measurement noise using limited state measurement and state estimate via a Kalman filter. The pilot experiments with the needle insertion robot were also conducted with the model assuming process and measurement noise and state estimate via a Kalman filter. The measurement model (29), the orthogonal distance from the desired plane, was obtained either through stereo triangulation of the needle tip from stereo cameras or as the orthogonal distance from the centerline of the C-arm imager. A semitransparent plastisol was used as a tissue surrogate. 


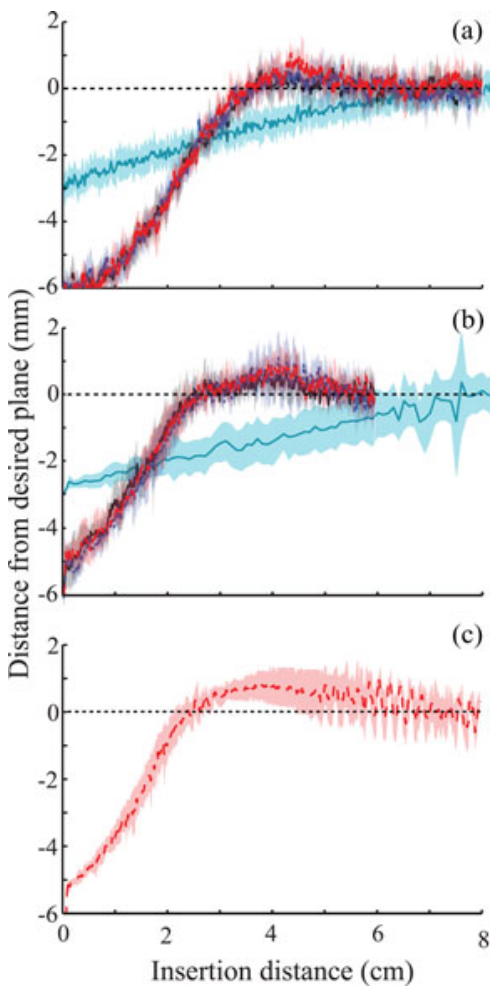

Fig. 2. Convergence plots for simulations, testbed experiments with stereo camera triangulation of needle tip, and experiments using C-arm fluoroscopy to track the needle tip. (a) Simulation results for $8-\mathrm{cm}$ insertions at $v=1 \mathrm{~mm} / \mathrm{s}$. The mean measurement of the tip distance from the plane (center line, solid or dashed) and standard deviation (shaded regions) for 1000 trials: estimation and control using 1 (red), 5 (blue), and 25 (black) modal states and using the torsional dynamics model to simulate the real dynamics and using no torsional information for feedback (turquoise). (b) Physical experiments for 6-cm insertions: estimation and control at $v=1 \mathrm{~mm} / \mathrm{s}$ using 1 (red), 5 (blue), and 25 (black) modal states shows categorical improvement over previously reported experimental control results (turquoise, adapted with permission from [18]]. (c) Physical experiments for $8-\mathrm{cm}$ insertions at $v=1 \mathrm{~mm} / \mathrm{s}$. These trials were conducted using only five modal states using C-arm fluoroscopy to track the needle tip distance from the desired plane. The mean trajectory and standard deviation for ten trials are shown, with an initial error of $6 \mathrm{~mm}$ from the desired plane.

In all simulations, we used a 25th-order torsional truncation as the "real" system. That is, the dynamical equations that were integrated to represent the true evolution of system state were based on the torsional dynamics that have 25 states. In reality, the system is infinite dimensional and not amenable to concrete computations in its complete representation. The choice of 25 states was limited by the amount of time required to integrate the system dynamics for the simulations. In both simulations and camera experiments, we show results associated with representing the torsional dynamics with 1,5 , and 25 truncated states and show that, for the particular parameters of our system, there is no difference between the number of modes chosen. This indicates that there is very little torsional windup inside of the tissue due to high damping and that the estimate of the first mode shape (dc mode) is sufficient to estimate the torsional windup outside the tissue. This is not always the case for different combinations of needles and tissues, as shown in [21] and [34]. The exact relationship between needle and tissue parameters that make torsional windup within the tissue relevant can be determined through the nondimensional analysis given in Appendix A.

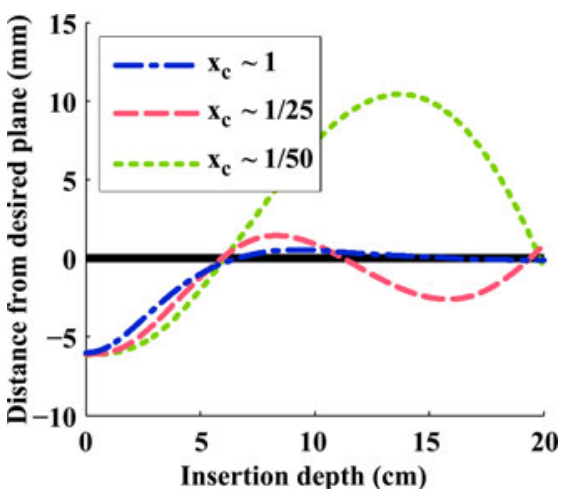

Fig. 3. Three different simulations of a needle insertion using a single torsional state and different characteristic lengths $x_{c}$ as computed using (17). As the characteristic length increases, more torsional states are needed to estimate and decouple the torsional dynamics from the kinematic motion of the needle tip. The characteristic length used to nondimensionalize the torsional dynamics is increased by either increasing the diameter of the needle, decreasing the needletissue damping, or combination of the two. An increase in the diameter of the needle will also increase the needle-tissue damping as viscous drag is a function of the normal force between the interacting materials.

\section{A. One Modal State is Sufficient for Control in Some Cases}

We conducted physical experiments with our needle insertion robot and artificial tissue (plastisol) in a laboratory environment. The experimental setup consisted of an industrial PC running a modified Ubuntu installation with RTAI real-time extensions (www.linuxcnc.org), stereo cameras for needle tip triangulation, and our needle insertion robot (the system is similar to that of Kallem and Cowan [18]). For these experiments, we performed a single insertion without rotating at the base and fit a circle to the recorded atip measurements to identify the radius of curvature. Values for viscous drag and shear modulus were obtained through parameter identification experiments using our needle diameter of $0.635 \mathrm{~mm}$. The viscous drag scales as a function of needle diameter and the polar moment of inertia scales as a function of the needle radius. The radius of curvature was determined experimentally by performing several insertions without twisting at the base and averaging the radius of curvature of circles fit to the needle trajectories.

Fig. 2(b) shows the results of five experiments for each model order. Experiments show very little difference between each of estimator/controller pairs based on the different model orders. Based on the nondimensional analysis shown in Appendix, this can be attributed to the following:

1) for short insertion distances with large portions of the needle outside the tissue, the dynamics are dominated by the windup in the portion outside the needle, and

2) for our combination of viscous drag and needle diameter, one modal state was able to capture the majority of torsional energy.

In our simulations, the total needle length is $L=30 \mathrm{~cm}$ and we are inserting at most $8 \mathrm{~cm}$. For the needle parameters given in Table I and this short insertion distance, by estimating a single-mode coefficient we are able to determine the difference between the angle at the base of the needle and the angle at the insertion point. According to the nondimensional analysis and the results of [21] and [34], a change in the needle geometry 
(a)

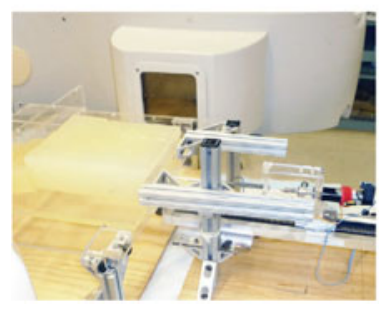

(b)

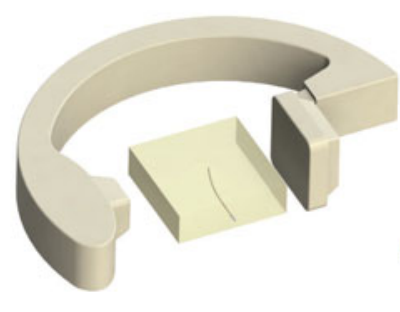

(c)

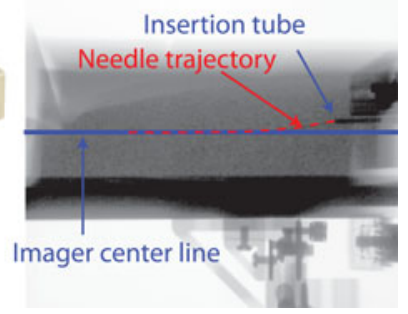

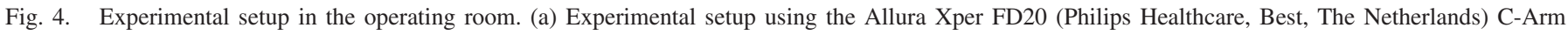

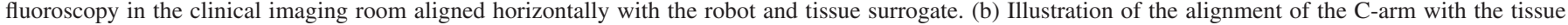

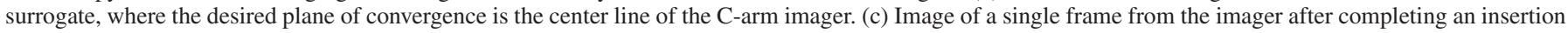
using continuous feedback control.

(length or diameter) or a change in tissue parameters (viscous damping) can necessitate the estimation of more mode shape coefficients. This is demonstrated by conducting simulations with the exact same needle parameters as those used for the physical experiments, as shown in Fig. 2(a). To show the effect of changing tissue or needle parameters, Fig. 3 shows how the rate of convergence changes when maintaining controller gains fixed and affecting a change in the characteristic length given in (17) either through a decrease in needle diameter, an increase in needle-tissue damping, or some combination of both. A corresponding increase in the magnitude of controller input could compensate for some of these dynamical effects, but control becomes fundamentally more difficult with a low number of modal state as characteristic length decreases.

\section{B. Simulations Using the Torsional Model Capture Behavior From Prior Experiments That Ignore Torsional Compliance}

In Fig. 2(a), results of simulations are shown using 1, 5, and 25 states for torsional compensation. Simulations that do not compensate for torsional dynamics, using the same controller presented in [18], are also shown. Our physical experiments conducted using the 1, 5, and 25 state torsional compensation and Kallem and Cowan's physical experiments without torsional compensation are shown in Fig. 2(b). The most salient observation is that the inclusion of torsional dynamics in the simulation model, coupled with a controller that does not compensate for the torsion, results in needle convergence behavior that closely matches that of the Kallem and Cowan experiments.

\section{C-Arm Fluoroscopy Experiments Indicate That Imaging Modality has Little Effect on the Efficacy of the Enhanced Model}

We conduct an experiment using a traditional clinical imaging modality, as opposed to stereo cameras with tip triangulation in transparent tissue surrogates, to evaluate the advanced torsional model in a clinical setting. Here, the image plane of the Allura Xper FD20 (Philips Healthcare, Best, The Netherlands) C-Arm fluoroscopy was aligned with the tissue surrogate such that the centerline of the image defined the plane in which we desired the needle to travel as shown in Fig. 4(a) and (b). The initial distance of the needle tip from the desire plane of travel was approximately $6 \mathrm{~cm}$. A typical postinsertion image is shown in Fig. 4(c), where the surrounding experimental apparatus was
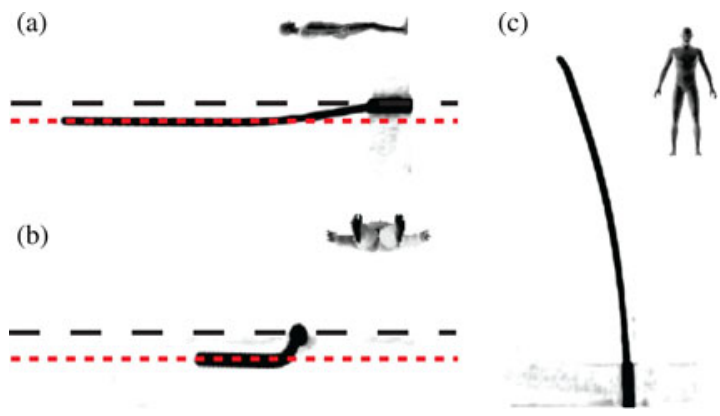

Fig. 5. Three axes imaging of the needle postinsertion using the Allura Xper FD20 (Philips Healthcare, Best, The Netherlands) C-Arm fluoroscopy for one of the trials. The images have been thresholded to facilitate tracking of the needle tip. (a) Side view of the needle after insertion. This is the view used during continuous insertion to track the needle tip and measure the distance from the center line of the $\mathrm{C}$-arm imager as the scaled orthogonal distance from the desired plane of travel. (b) End-on view of the needle after insertion. (c) Top view of the needle curving inside the tissue. In a and b, the long-dashed line indicates the needle start position at a distance of $6 \mathrm{~mm}$ from the desired plane and the short-dashed line indicates the plane to which we desire to control.

cropped to facilitate the thresholding of the image for needle tip tracking. Fig. 5 shows the final result of a typical insertion along three axes with the desired plane of convergence indicated with the dashed line. Here, the image was thresholded to facilitate both needle tip tracking and to remove artifacts of the surrounding tissue from the image.

From Fig. 2(c), we observed that when moving from the stereo cameras to the fluoroscopy imaging that the convergence results were nearly identical. The approach of defining the center line of the imager as a means of defining a desired plane of travel introduces a new method of set-point control. By reorienting the plane, either automatically through planning software or manually by a clinician, and ensuring the needle tip is tangent to the plane both before and after reorientation, needle trajectories can be defined via consecutive planes, without stereo imaging. This method of allowing a clinician or computer planner to define successive planes of travel facilitates continuous insertions, where the needle is only stopped when the plane needs to be reoriented.

A comprehensive review of dosages for common medical procedures with radiative imaging can be found in the three part series [36]-[38] and an earlier study on a broad range of interventional procedures [39]. The latter determined the radiation 
doses for common procedures to lie mostly between 24.2 and $74.7 \mathrm{~Gy} \cdot \mathrm{cm}^{2}$. Our dosage rate was $338 \mathrm{mGy} \cdot \mathrm{cm}^{2} / 60 \mathrm{~s}$ of insertion, and each insertion was done at a cautious rate taking approximately 90 s/insertion. This dosage was based on a standard clinical protocol provided by the clinical hardware for peripheral scans of arms or legs for deep vein thrombosis treatments, with an adjustment from 3 to 15 frames/s. This dosage lies well within the bounds of acceptable dosage limits, even if used in an area of the body that require higher output power and multiple insertions.

\section{CONCLUSION}

We presented a new model for torsional dynamics, which takes into account time-varying boundary conditions. The primary conclusion from our experimental results is that the incorporation of these time-varying dynamics into a closed-loop control scheme significantly improves performance over the purely kinematic control method used previously. Specifically, the experimental trials reported in Kallem and Cowan [18], which did not incorporate torsional dynamics, required an insertion distance of about $8 \mathrm{~cm}$ to recover from a $3-\mathrm{mm}$ initial error from the desired plane. Here, we show experimental convergence to the plane in approximately $2.5-3.0 \mathrm{~cm}$ of insertion despite a larger initial error from the plane of $6 \mathrm{~mm}$.

The proposed model of torsional dynamic and needle motion has shown work in simulation, testbed experiments with stereo camera in semitransparent tissue surrogates, and using $\mathrm{C}$-arm fluoroscopy imaging. The efficacy of the model in multiple methods of imaging points to the general effectiveness of a dynamical torsion model as needle steering moves toward clinical use. Herein, our measurement model was the tip position of the needle. A more rich measurement, e.g., a triangulation of the entire needle shape via biplane fluoroscopy or MRI or a magnetic tracker, would provide even more accurate estimates of torsional states and improve both estimation and control.

Interestingly, the physical experiments showed a faster than expected convergence for the model with a low number of torsional states. The model order did not seem to have a great impact on the rate of convergence for the physical experiments. This was due to the relatively high damping and small needle size, thus concentrating the torsion of the needle to the portion of the needle outside the tissue. This presents the need to develop needle insertion robots for tip-steerable needles that can control the rotation of the needle at the point of insertion, as opposed to the insertion robot presented herein requiring a large portion outside the tissue.

Finally, the use of C-arm fluoroscopy as a feedback control sensing modality was just as effective as tip triangulation through stereo feedback. Using the center line of the imager to define the desired plane of travel suggest a potential method of defining trajectory setpoint control through reorientation of the imager, either manually by a clinician or through software planning methods. Additionally, the radiation dosages used to complete the needle insertions were well within bound of existing fluoroscopy-based procedures, further validating the feasibility of using automatic, fluoroscopy-based feedback control.
TABLE II

DIMENSIONAL VARIABLES IN THE PDE DESCRIBING NEEDLE TORSION

\begin{tabular}{lcc}
\hline \hline Description & Symbol & Dimensions \\
\hline Moment of inertia & $\eta(\mathrm{kgm})$ & $M L$ \\
Damping & $\beta\left(\frac{\mathrm{kmm}_{\mathrm{s}}}{\mathrm{s} 3}\right)$ & $M L T^{-1}$ \\
Stiffnesss & $\kappa\left(\frac{\mathrm{kgm}^{3}}{\mathrm{~s}^{2}}\right)$ & $M L^{3} T^{-1}$ \\
Input torque & $\tau\left(\frac{\mathrm{kg} \mathrm{m}^{2}}{\mathrm{~s}^{2}}\right)$ & $M L^{2} T^{-1}$ \\
Time & $t(\mathrm{~s})$ & $T$ \\
Point along the needle & $x(\mathrm{~m})$ & $L$ \\
Needle angle & $\theta$ (unitless radians) & 1 \\
\hline \hline
\end{tabular}

\section{APPENDIX \\ NONDIMENSIONALIZATION OF TORSIONAL DYNAMICS OF TIP-STEERABLE NEEDLES}

By nondimensionalizing the PDE representing the torsional dynamics of the needle, we can gain insight into the relevant parameters of the system. It results in three characteristic variables that give relative importance into the behavior of the system as a function of the parameters of the system (shear modulus, polar moment of inertia, density, etc.).

\section{A. Derivation}

We begin with the nonseparable form of the PDE describing the torsion along the needle as a function of time given in (3), then list all of the relevant dependent and independent variables of the system in Table II and proceed to determine the $\Pi$-variables that nondimensionalize the system.

We find four $\Pi$-groups describing a change of coordinates that nondimensionalizes the original system state, namely

$$
x=\bar{x} \underbrace{\sqrt{\frac{\eta \kappa}{\beta^{2}}}}_{x_{c}}, \quad t=\bar{t} \underbrace{\frac{\eta}{\beta}}_{t_{c}} \quad \tau=\bar{\tau} \underbrace{\sqrt{\frac{\beta^{2} \kappa}{\eta}}}_{\tau_{c}}
$$

with dimensionless variables $\bar{\tau}, \bar{x}$, and $\bar{t}$. Then, we derive the differentials

$$
\begin{gathered}
d t=t_{c} d \bar{t} \\
d x=x_{c} d \bar{x} .
\end{gathered}
$$

Using the chain rule and the relationships between differentials, we then substitute back into the original PDE

$$
\eta \frac{1}{t_{c}^{2}} \frac{\partial^{2} \theta}{\partial \bar{t}^{2}}+\beta \frac{1}{t_{c}} \frac{\partial \theta}{\partial \bar{t}}-\kappa \frac{1}{x_{c}^{2}} \frac{\partial^{2} \theta}{\partial \bar{x}^{2}}=\delta\left(x_{c} \bar{x}\right) \tau_{c} \bar{\tau} .
$$

Divide through by the first coefficient

$$
\frac{\partial^{2} \theta}{\partial \bar{t}^{2}}+\frac{\beta}{\eta} t_{c} \frac{\partial \theta}{\partial \bar{t}}-\frac{\kappa}{\eta} \frac{t_{c}^{2}}{x_{c}^{2}} \frac{\partial^{2} \theta}{\partial \bar{x}^{2}}=\delta\left(x_{c} \bar{x}\right) \frac{\tau_{c} t_{c}^{2}}{\eta} \bar{\tau}
$$

then substitute $t_{c}, x_{c}$, and $\tau_{c}$ from aforesaid, such that

$$
\frac{\partial^{2} \theta}{\partial \bar{t}^{2}}+\frac{\partial \theta}{\partial \bar{t}}-\frac{\partial^{2} \theta}{\partial \bar{x}^{2}}=\delta\left(x_{c} \bar{x}\right) x_{c} \bar{\tau} .
$$




\section{B. Characteristic Variables}

The shear modulus $G$ and the density of Nitinol $\rho$ is fairly constant across both manufacturers and sizes of the types of superelastic Nitinol wires we use as needles. The polar moment of inertia of a needle is related to the diameter of the needle to the fourth power as $J=\frac{\pi d^{4}}{32}$. We rewrite the characteristic variables and include the parameters of the system that constitute the inertia and stiffness

$$
\begin{aligned}
& x_{c}=\sqrt{\frac{\eta \kappa}{\beta^{2}}}=\frac{J}{\beta} \sqrt{\rho G}=\frac{d^{4}}{\beta}\left(\frac{\pi}{32} \sqrt{\rho G}\right) \sim \frac{d^{4}}{\beta} \\
& t_{c}=\frac{\eta}{\beta}=\frac{\rho J}{\beta}=\frac{d^{4}}{\beta} \frac{\rho \pi}{32} \sim \frac{d^{4}}{\beta} \\
& \tau_{c}=\sqrt{\frac{\beta^{2} \kappa}{\eta}}=\beta \sqrt{\frac{J G}{\rho J}}=\beta \sqrt{\frac{G}{\rho}} \sim \beta .
\end{aligned}
$$

The three characteristic variables each tell us about the behavior of the system.

1) The characteristic variable $t_{c}$ tells us that the time period of oscillations in the system varies proportional to the needle diameter and inversely proportional to the needle-tissue damping.

2) The characteristic variable $x_{c}$ tells us that the spatial period of oscillations in the system varies proportional to the needle diameter and inversely proportional to the needletissue damping.

3) The characteristic variable $\tau_{c}$ tells us that the magnitude of required torque input increases proportional to the needletissue damping.

These characteristics variables end up showing exactly what may be intuitive to some, namely that 1) as damping increases then oscillations increase, 2) as needle diameter decreases (i.e., stiffness and inertia decrease) then oscillations increase, and 3) as damping increases then requisite torque inputs also increases. Also, an important aspect of needle diameter is that a larger needle diameter will displace a larger amount of tissue, creating a larger normal force along the needle, thus creating increases in the viscous damping coefficient that increases proportional to the needle diameter.

\section{ACKNOWLEDGMENT}

The authors thank collaborators from the JHU needle steering group for their contributions and discussions: Dr. T. Wedlick, A. Majewicz, Dr. K. Reed, Dr. G. Chirikjian, Dr. W. Park, and Dr. S. Misra.

\section{REFERENCES}

[1] A. Wood, "New method of treating neuralgia by the direct application of opiates to the painful points," Edin. Med. Surg. J., vol. 82, pp. 265-281, 1855.

[2] Y. Nakajima, T. Dohi, T. Sasama, Y. Momoi, N. Sugano, Y. Tamura, S. hwan Lim, I. Sakuma, M. Mitsuishi, T. Koyama, K. Yonenobu, S. Ohashi, M. Bessho, and I. Ohnishi, "Surgical tool alignment guidance by drawing two cross-sectional laser-beam planes," IEEE Trans. Biomed. Eng., vol. 60, no. 6, pp. 1467-1476, Jun. 2013.

[3] K. Cleary, M. Clifford, D. Stoianovici, M. Freedman, S. K. Mun, an V. Watson, "Technology improvements for image-guided and minimally invasive spine procedures," IEEE Trans. Inf. Technol. Biomed., vol. 6 , no. 4, pp. 249-261, Dec. 2002

[4] S. DiMaio and S. Salcudean, "Needle insertion modeling and simulation," IEEE Trans. Robot. Automat., vol. 19, no. 5, pp. 864-875, Oct. 2003

[5] S. Okazawa, R. Ebrahimi, J. Chuang, S. E. Salcudean, and R. Rohling, "Hand-held steerable needle device," IEEE/ASME Trans. Mechatronics, vol. 10, no. 3, pp. 285-296, Jun. 2005.

[6] R. J. Webster III, J. S. Kim, N. J. Cowan, G. S. Chirikjian, and A. M. Okamura. (2006, May). Nonholonomic modeling of needle steering. Int. J. Robot. Res. [Online]. 25(5/6), pp. 509-526. Available: http://dx.doi.org/10.1177/0278364906065388

[7] P. E. Dupont, J. Lock, B. Itkowitz, and E. Butler, "Design and control of concentric-tube robots," IEEE Trans. Robot., vol. 26, no. 2, pp. 209-225, Apr. 2010.

[8] H. Takaki, K. Yamakado, A. Nakatsuka, T. Yamada, J. Uraki, M. Kashima, T. Yamanaka, K. Shiraki, Y. Takei, and K. Takeda, "Computed tomography fluoroscopy-guided radiofrequency ablation following intra-arterial iodized-oil injection for hepatocellular carcinomas invisible on ultrasonographic images," Int. J. Clin. Oncol., vol. 18, no. 1, pp. 46-53, Feb. 2013.

[9] S. Akhlaghpoor, F. Dahi, M. Alinaghizadeh, and A. A. Shabestari, "CT fluoroscopy-guided transcaval radiofrequency ablation of insulinoma," J. Vascular Interventional Radiol., vol. 22, no. 3, pp. 409-410, Mar. 2011.

[10] M. Lutze, R. Stendel, J. Vesper, and M. Brock, "Periradicular therapy in lumbar radicular syndromes: Methodology and results," Acta Neurochir (Wien), vol. 139, no. 8, pp. 719-724, 1997.

[11] R. A. Omary, J. A. Gehl, B. E. Schirf, J. D. Green, B. Lu, F. S. Pereles, J. Huang, A. C. Larson, and D. Li, "MR imaging- versus conventional $\mathrm{X}$-ray fluoroscopy-guided renal angioplasty in swine: Prospective randomized comparison," Radiology, vol. 238, no. 2, pp. 489-496, Feb. 2006.

[12] S. G. Kroeze, M. Huisman, H. M. Verkooijen, P. J. van Diest, J. L. Ruud Bosch, and M. A. van den Bosch, "Real-time 3D fluoroscopy-guided large core needle biopsy of renal masses: A critical early evaluation according to the IDEAL recommendations," Cardiovascular Interventional Radiol., vol. 35 , no. 3, pp. 680-685, Jun. 2012.

[13] A. Hoznek, I. Ouzaid, M. Gettman, J. Rode, A. De La Taille, L. Salomon, and C. C. Abbou, "Fluoroscopy-guided renal access in supine percutaneous nephrolithotomy," Urology, vol. 78, no. 1, pp. 221-224, Jul. 2011.

[14] A. Marien, I. Gill, O. Ukimura, B. Nacim, and A. Villers, "Target ablationImage-guided therapy in prostate cancer," Urol. Oncol., Jan. 2014.

[15] B. J. Kim, J. W. Lee, S. J. Kim, G. Y. Lee, and H. S. Kang, "Diagnostic yield of fluoroscopy-guided biopsy for infectious spondylitis," AJNR Amer. J. Neuroradiol., vol. 34, no. 1, pp. 233-238, Jan. 2013.

[16] A. Muacevic and A. Muller, "Image-guided endoscopic ventriculostomy with a new frameless armless neuronavigation system," Comput. Aided Surg., vol. 4, no. 2, pp. 87-92, 1999.

[17] W. Park, J. S. Kim, Y. Zhou, N. J. Cowan, A. M. Okamura, and G. S. Chirikjian, "Diffusion-based motion planning for a nonholonomic flexible needle model," in Proc. IEEE Int. Conf. Robot. Autom., Apr. 2005 pp. 4600-4605.

[18] V. Kallem and N. J. Cowan. (2009, Feb.). Image guidance of flexible tip-steerable needles. IEEE Trans. Robot. [Online]. 25(1), pp. 191-196. Available: http://dx.doi.org/10.1109/TRO.2008.2010357

[19] J. A. Engh, D. S. Minhas, D. Kondziolka, and C. N. Riviere, "Percutaneous intracerebral navigation by duty-cycled spinning of flexible bevel-tipped needles," Neurosurgery, vol. 67, no. 4, pp. 1117-1123, 2010.

[20] M. Bernardes, B. Adorno, P. Poignet, and G. Borges, "Robot-assisted automatic insertion of steerable needles with closed-loop imaging feedback and intraoperative trajectory replanning," Mechatronics, vol. 23, pp. 630-645, 2013.

[21] K. B. Reed, A. M. Okamura, and N. J. Cowan. (2009, Dec.). Modeling and control of needles with torsional friction. IEEE Trans. Biomed. Eng. [Online]. 56(12), pp. 2905-2916. Available: http://dx.doi.org/10.1109/TBME.2009.2029240

[22] T. Wedlick and A. Okamura, "Characterization of pre-curved needles for steering in tissue," in Proc. IEEE Annu. Int. Conf. Eng. Med. Biol. Soc., 2009, pp. 1200-1203

[23] D. Glozman and M. Shoham, "Image-guided robotic flexible needle steering," IEEE Trans. Robot., vol. 23, no. 3, pp. 459-467, Jun. 2007.

[24] V. G. Mallapragada, N. Sarkar, and T. K. Podder, "Robot-assisted realtime tumor manipulation for breast biopsy," IEEE Trans. Robot., vol. 25, no. 2, pp. 316-324, Apr. 2009. 
[25] K. B. Reed, A. Majewicz, V. Kallem, R. Alterovitz, K. Goldberg, N. J. Cowan, and A. M. Okamura, "Robot-assisted needle steering," IEEE Robot. Autom. Mag., vol. 18, no. 4, pp. 35-46, Dec. 2011.

[26] K. B. Reed, V. Kallem, R. Alterovitz, K. Goldberg, A. M. Okamura, and N. J. Cowan, "Integrated planning and image-guided control for planar needle-steering," in Proc. IEEE Int. Conf. Biomed. Robot. Biomechatronics, Scottsdale, AZ, USA, Oct. 2008, pp. 819-824.

[27] M. Abayazid, R. Roesthuis, R. Reilink, and S. Misra, "Integrating deflection models and image feedback for real-time flexible needle steering," IEEE Trans. Robot., vol. 29, no. 2, pp. 542-553, Apr. 2013.

[28] G. S. Fischer, I. Iordachita, C. Csoma, J. Tokuda, S. P. DiMaio, C. M. Tempany, N. Hata, and G. Fichtinger, "MRI-compatible pneumatic robot for transperineal prostate needle placement," IEEE/ASME Trans. Mechatronics, vol. 13, no. 3, pp. 295-305, Jun. 2008.

[29] A. Krieger, R. C. Susil, C. Ménard, J. A. Coleman, G. Fichtinger, E. Atalar, and L. L. Whitcomb, "Design of a novel MRI compatible manipulator for image guided prostate interventions," IEEE Trans. Biomed. Eng., vol. 52, no. 2, pp. 306-313, Feb. 2005.

[30] R. Seifabadi, S.-E. Song, A. Krieger, N. B. Cho, J. Tokuda, G. Fichtinger, and I. Iordachita, "Robotic system for MRI-guided prostate biopsy: feasibility of teleoperated needle insertion and ex vivo phantom study," Int. J. Comput. Assisted Radiol. Surg., vol. 7, no. 2, pp. 181-190, 2012.

[31] P. Holmes, J. Lumley, and G. Berkooz, Turbulence, Coherent Structures, Dynamical Systems and Symmetry. Cambridge, U.K.: Cambridge Univ. Press, 1998.

[32] K. Sze, S. Chen, and J. Huang, "The incremental harmonic balance method for nonlinear vibration of axially moving beams," J. Sound Vibration, vol. 281, no. 3-5, pp. 611-626, 2005.

[33] L. Wang, Z. Hu, Z. Zhong, and J. Ju, "Hamiltonian dynamic analysis of an axially translating beam featuring time-variant velocity," Acta Mechanica, vol. 206, no. 3, pp. 149-161, 2009.

[34] J. P. Swensen and N. J. Cowan, "Torsional dynamics compensation enhances robotic control of tip-steerable needles," in Proc. IEEE Int. Conf. Robot. Autom., May 2012, pp. 1601-1606.

[35] W. Park, K. B. Reed, A. M. Okamura, and G. S. Chirikjian, "Estimation of model parameters for steerable needles," in Proc. IEEE Int. Conf. Robot. Autom., May 2010, pp. 3703 -3708.

[36] D. L. Miller, S. Balter, P. E. Cole, H. T. Lu, B. A. Schueler, M. Geisinger, A. Berenstein, R. Albert, J. D. Georgia, P. T. Noonan, J. F. Cardella, J. S. George, E. J. Russell, T. W. Malisch, R. L. Vogelzang, G. L. Miller III, and J. Anderson, "Radiation doses in interventional radiology procedures: The RAD-IR study part I: Overall measures of dose," J. Vascular Interventional Radiol., vol. 14, no. 6, pp. 711-727, 2003.

[37] D. L. Miller, S. Balter, P. E. Cole, H. T. Lu, A. Berenstein, R. Albert, B. A. Schueler, J. D. Georgia, P. T. Noonan, E. J. Russell, T. W. Malisch, R. L. Vogelzang, M. Geisinger, J. F. Cardella, J. S. George, G. L. Miller, and J. Anderson, "Radiation doses in interventional radiology procedures: The RAD-IR study part II: Skin dose," J. Vascular Interventional Radiol., vol. 14, no. 8, pp. 977-990, 2003.

[38] S. Balter, B. A. Schueler, D. L. Miller, P. E. Cole, H. T. Lu, A. Berenstein, R. Albert, J. D. Georgia, P. T. Noonan, E. J. Russell et al., "Radiation doses in interventional radiology procedures: The RAD-IR study: Part III: Dosimetric performance of the interventional fluoroscopy units," J. Vascular Interventional Radiol., vol. 15, no. 9, pp. 919-926, 2004.

[39] B. J. McParland. (1998). A study of patient radiation doses in interventional radiological procedures. Brit. J. Radiol. [Online]. 71(842), pp. 175-85. Available: http://bjr.birjournals.org/content/71/842/175. abstract

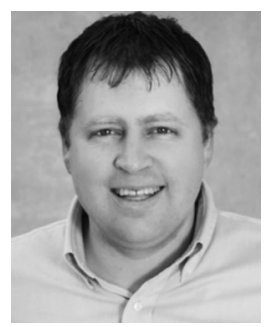

John P. Swensen (S'00-M'12) received the B.S. degree in electrical engineering from Utah State University, Logan, UT, USA, in 2003, and M.S. and $\mathrm{Ph} . \mathrm{D}$. degrees in mechanical engineering from Johns Hopkins University, Baltimore, MD, USA, in 2009 and 2012, respectively.

In 2011, he joined the GRAB Lab at Yale University, New Haven, CT, USA, as a Postdoctoral Associate. His research interests include medical robotics, geometrics estimation and control, and robotic multifunctional materials.

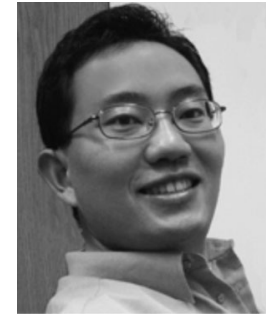

MingDe Lin (M'07) received the B.S. degree from Rensselaer Polytechnic Institute, Troy, NY, USA, in 2001, and the Ph.D. degree from Duke University, Durham, NC, USA, in 2008, all in biomedical engineering.

$\mathrm{He}$ is currently a Clinical Site Senior Researcher for Philips Research North America, Briarcliff Manor, NY, USA, and has been stationed at Johns Hopkins Hospital, Baltimore, MD, USA, since 2008 to collaborate with clinicians in developing X-ray imaging systems for interventional radiology/oncology. His research focus is in improving image quality, providing image-guided navigation tools, and quantifying therapy success in the area of hepatic carcinoma (liver cancer) treatment using transcatheter arterial chemoembolization.

Dr. Lin is the Principle Investigator for a National Cancer Institute grant for this work titled "See, Reach, Treat, Tumor - Optimized Transarterial Chemoembolization Drug Delivery (R01 CA160771)".

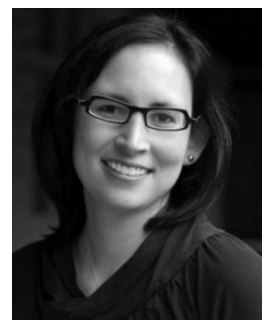

Allison M. Okamura (S'98-A'00-M'03-SM'09F'11) received the B.S. degree from the University of California, Berkeley, Berkeley, CA, USA, in 1994 , and the M.S. and Ph.D. degrees from Stanford University, Stanford, CA, in 1996 and 2000, respectively, all in mechanical engineering.

She is currently an Associate Professor of mechanical engineering at Stanford University, where she is also the Robert Bosch Faculty Scholar. She was previously a Professor and the Vice Chair of mechanical engineering at Johns Hopkins University, Baltimore, MD, USA. Her research interests include haptics, teleoperation, medical robotics, virtual environments and simulation, neuromechanics and rehabilitation, prosthetics, and engineering education.

Dr. Okamura received the 2004 National Science Foundation CAREER Award, the 2005 IEEE Robotics and Automation Society Early Academic Career Award, and the 2009 IEEE Technical Committee on Haptics Early Career Award. She is an Associate Editor of the IEEE TRANSACTIONS ON HAPTICS.

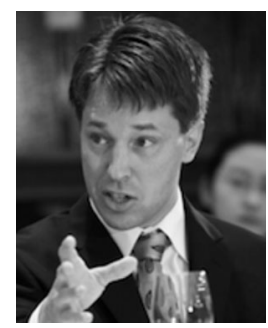

Noah J. Cowan (S'99-M'01-SM'12) received the B.S. degree from the Ohio State University, Columbus, OH, USA, in 1995, and the M.S. and $\mathrm{Ph}$.D. degrees from the University of Michigan, Ann Arbor, MI, USA, in 1997 and 2001, respectively, all in electrical engineering.

From 2001 to 2003, he was a Postdoctoral Fellow in integrative biology at the University of California, Oakland, CA, USA. In 2003, he joined the Department of Mechanical Engineering at Johns Hopkins University, Baltimore, MD, USA, where he is currently an Associate Professor. His research interests include mechanics and multisensory control in animals and machines.

Dr. Cowan received the National Science Foundation PECASE Award in 2010, the James S. McDonnell Foundation Scholar Award in Complex Systems in 2012, and the William H. Huggins Award for excellence in teaching in 2004 and a Rackham Doctoral Fellowship from the University of Michigan in 2000. 